\title{
A novel adaptive time stepping variant of the Boris-Buneman integrator for the simulation of particle accelerators with space charge
}

\author{
Matthias Toggweiler ${ }^{\mathrm{a}, \mathrm{b}, \mathrm{c}}$, Andreas Adelmann ${ }^{\mathrm{b}, *}$, Peter Arbenz ${ }^{\mathrm{a}}$, Jianjun J. Yang ${ }^{\mathrm{b}}$ \\ ${ }^{a}$ ETH Zürich, Computer Science Department, Universitätsstrasse 6, 8092 Zürich, Switzerland \\ ${ }^{b}$ Paul Scherrer Institute, CH-5234 Villigen, Switzerland \\ ${ }^{c}$ MIT, Department of Physics, 77 Massachusetts Avenue, MA 02139, United States
}

\begin{abstract}
We show that adaptive time stepping in particle accelerator simulation is an enhancement for certain problems. The new algorithm has been implemented in the OPAL (Object Oriented Parallel Accelerator Library) framework. The idea is to adjust the frequency of costly self field calculations, which are needed to model Coulomb interaction (space charge) effects. In analogy to a Kepler orbit simulation that requires a higher time step resolution at the close encounter, we propose to choose the time step based on the magnitude of the space charge forces. Inspired by geometric integration techniques, our algorithm chooses the time step proportional to a function of the current phase space state instead of calculating a local error estimate like a conventional adaptive procedure. Building on recent work, a more profound argument is given on how exactly the time step should be chosen. An intermediate algorithm, initially built to allow a clearer analysis by introducing separate time steps for external field and self field integration, turned out to be useful by its own, for a large class of problems.
\end{abstract}

Keywords: Adaptive time stepping, Boris-Buneman, particle-in-cell, space charge, particle accelerator simulation

\section{Introduction}

In recent years, precise beam dynamics simulations in the design of high-current low-energy hadron machines as well as of 4th generation light sources have become a very important research topic. Hadron machines are characterized by high currents and hence require excellent control of beam losses and/or keeping the emittance (a measure of the phase space) of the beam in narrow ranges. This is a challenging problem which requires the accurate modeling of the dynamics of a large ensemble of macro or real particles subject to complicated external focusing, accelerating and wake-fields, as well as the self-fields caused by the Coulomb interaction of the particles.

The simulation method discussed in this paper is part of a general accelerator modeling tool, OPAL (Object Oriented Parallel Accelerator Library) [1]. OPAL allows to tackle the most challenging problems in the field of high precision particle accelerator modeling. These include the simulation of high power hadron accelerators and of next generation light sources. Recent physics proposals include [2-4], all of them require large scale particles based simulation in order to design and optimize the required high power hadron machines.

Here, we discuss methods which track the orbit of each particle individually, with time as the independent variable. Accurate modeling demands the usage of many simulation particles,

\footnotetext{
${ }^{*}$ Corresponding author

Email addresses: $\mathrm{rmf} 7 \mathrm{0m} 4 \mathrm{t} . \mathrm{ch}$ (Matthias Toggweiler), andreas .adelmann@psi.ch (Andreas Adelmann), arbenz@inf .ethz.ch (Peter Arbenz), jianjun.yang@psi.ch (Jianjun J. Yang)
}

which of course makes this approach expensive. The flow of a particle is described as an initial value problem for a differential equation, therefore such methods are called (time) integrators. What is common to them is that they create a discrete trajectory that approximates the solution of the initial value problem. How they transport a given state from time $t_{n}$ to $t_{n+1}$ is crucial for accuracy and computational effort.

Currently, only time integrators that use constant time steps $\Delta t=t_{n+1}-t_{n}$ are utilized in OPAL. The goal of the recent master thesis [5] was to investigate whether an integrator that uses variable time steps can provide enhanced efficiency. Since the space charge solver, which computes the self field induced by the charge, requires all particles to be synchronized in time, there is a global time step shared among all the particles. Only this global time step is to be adapted. Other kinds of adaptation, like adaptive mesh refinement, are of separate concern and not the subject of this work.

In [5] two categories of adaptive time stepping schemes were identified:

(a) conventional adaptivity that modifies the time step based on a local error estimate, and

(b) geometric adaptive integration which aims at solving a regularized differential equation.

Both categories adapt the time step based on the local dynamics. However, the way how this is achieved differs. In (a), the step size is changed such that a predicted local error is below some target value, assuming that the local error is roughly proportional to some power of the step size. The local error estimate is usually obtained by comparing two local solutions of 
different order, like, e.g., in Runge-Kutta schemes with step size control [6]. Typical for this kind of algorithms is that a step can be rejected and repeated if the new local error estimate is above the tolerance. On the other hand, the origin of (b), geometric adaptive integration, are the deficiencies of (a) when applied to systems with special structure, e.g. Hamiltonian systems. Integrators, made adaptive in the conventional way, lose their reversibility or symplecticity properties.

First, it was not clear, how to obtain an appropriate and cheap-to-compute local error estimate in our particle-in-cell application for approach (a), and the possibility that steps have to be rejected and repeated indicated that the memory consumption may increase. The reason why we considered approach (b) was the hope that it would present a light-weight alternative with less overhead. Strict time-reversibility of the adaptive scheme was not a major issue.

A Kepler orbit problem was chosen as a model problem of adaptive geometric integration. A leapfrog method, adaptive in the sense of (b), was used to perform smaller steps when the comet is near the sun and attracted by large forces, and larger steps when the comet is far away. Following this model, we derived an adaptive variant of the Boris-Buneman method. Where in the Kepler case we adapted the time step to the strength of the gravity force, in our application it is adapted to the strength of space charge forces. In both cases, the strength of the force is proportional to the square of the distance, just the sign and the coupling constant is different for the Coulomb force.

A first implementation proved this type of adaptive time stepping to be beneficial for particle accelerator simulations, see the results on two photoinjector scenarios in [5]. However, a clear argument was missing on how the time step should be chosen for optimal results. Moreover, time step requirements for external fields were not considered. In this follow-up work, based on a 1D model problem, we give a sound motivation for the used adaptation scheme. The enhanced version of the adaptive algorithm still chooses the time step based on space charge forces only, but allows finer inner time steps in a multiple-timestepping fashion to account for fast varying external fields.

The initial hope was that we can show the usefulness of variable time steps for many other scenarios too. However, the benefit over constant steps with our algorithm becomes visible only when variations in space charge forces are large. This is the case for space charge-dominated scenarios like a photoinjector simulation, but e.g. not for simulations of high-energy cyclotron turns. For cases where variations in the space charge forces are small but space charge still has a visible effect, the multiple-time-stepping extension alone proved to be an important enhancement to the original Boris-Buneman method. Although it was thought to be only an intermediate algorithm and its derivation was relatively easy compared to the rest, this part is probably the achievement with broader practical impact.

In Section 2 we state the problem. In Section 3 follows an introduction to the current time integration scheme available in OPAL. The new adaptive Boris-Buneman scheme is presented in Section 4 building on an multiple-time-stepping extension of the original method. In Section 5 we examine more closely the adaptive step size strategy by looking at a one-dimensional model problem. In Section 6 we benchmark the developed methods on applications from beam dynamics, and in Section 7 we draw our conclusions.

\section{Particle beam simulations with space charge}

\subsection{The electrostatic particle-in-cell method}

We consider the Vlasov-Poisson description of the phase space, including external and self fields. Let $f(\mathbf{x}, \mathbf{v}, t)$ be the density of the particles in the phase space, i.e. the positionvelocity $(\mathbf{x}, \mathbf{v})$ space. Its evolution is determined by the collisionless Vlasov equation,

$$
\frac{d f}{d t}=\partial_{t} f+\mathbf{v} \cdot \nabla_{\mathbf{x}} f+\frac{q}{m}(\mathbf{e}+\mathbf{v} \times \mathbf{b}) \cdot \nabla_{\mathbf{v}} f=0,
$$

where $m, q$ denote particle mass and charge, respectively. The electric and magnetic fields $\mathbf{e}$ and $\mathbf{b}$ are superpositions of external fields and self fields (space charge),

$$
\mathbf{e}=\mathbf{e}^{\text {ext }}+\mathbf{e}^{\text {self }}, \quad \mathbf{b}=\mathbf{b}^{\text {ext }}+\mathbf{b}^{\text {self }}
$$

If $\mathbf{e}$ and $\mathbf{b}$ are known, then each particle can be propagated according to the equation of motion for charged particles in an electromagnetic field. After particles have moved, we have to update $\mathbf{e}^{\text {self }}$ and $\mathbf{b}^{\text {self }}$ (among other things). For this, we change the coordinate system into the one moving with the particles. By means of the appropriate Lorentz transformation $\mathcal{L}$ [7] we arrive at a (quasi-) static approximation of the system in which the transformed magnetic field becomes negligible, $\hat{\mathbf{b}} \approx \mathbf{0}$. The transformed electric field is then obtained from

$$
\hat{\mathbf{e}}=\hat{\mathbf{e}}^{\text {self }}=-\nabla \hat{\phi}
$$

where the electrostatic potential $\hat{\phi}$ is the solution of the Poisson problem

$$
-\Delta \hat{\phi}(\mathbf{x})=\frac{\mathcal{L}(\rho(\mathbf{x}))}{\varepsilon_{0}},
$$

equipped with appropriate boundary conditions. Here, $\rho$ denotes the spatial charge density and $\varepsilon_{0}$ is the dielectric constant. By means of the inverse Lorentz transformation $\left(\mathcal{L}^{-1}\right)$ the electric field $\hat{\mathbf{e}}$ can then be transformed back to yield both the electric and the magnetic fields in (1).

The Poisson problem (3) discretized by finite differences can efficiently be solved on a rectangular grid by a ParticleIn-Cell (PIC) approach [8]. The right hand side of (3) is discretized by sampling the particles at the grid points. In (2), $\hat{\phi}$ is interpolated at the particle positions from its values at the grid points. We also note that the FFT-based Poisson solvers and similar approaches [8, 9] are most effective in box-shaped or open domains.

\subsection{Equations of motion}

We integrate in time $N$ identical particles, all having the rest mass $m$ and charge $q$. The relativistic equations of motion for 
particle $i$ are

$$
\begin{aligned}
\frac{\mathrm{d} \mathbf{x}_{i}}{\mathrm{~d} t} & =\frac{\mathbf{p}_{i}}{m \gamma_{i}}, \\
\frac{\mathrm{d} \mathbf{p}_{i}}{\mathrm{~d} t} & =q\left(\mathbf{e}_{i}+\frac{\mathbf{p}_{i}}{m \gamma_{i}} \times \mathbf{b}_{i}\right),
\end{aligned}
$$

where $\mathbf{x}_{i}$ is the position, $\mathbf{p}_{i}=m \mathbf{v}_{i} \gamma_{i}$ the relativistic momentum, $\mathbf{v}_{i}$ the velocity, $\gamma_{i}=1 / \sqrt{1-\left(\left\|\mathbf{v}_{i}\right\| / c\right)^{2}}=\sqrt{1+\left(\left\|\mathbf{p}_{i}\right\| /(m c)\right)^{2}}$ the Lorentz factor and $c$ the speed of light. The electric and magnetic field, $\mathbf{e}_{i}$ and $\mathbf{b}_{i}$, can be decomposed into external field and self field contributions:

$$
\begin{aligned}
\mathbf{e}_{i} & =\mathbf{e}^{\text {ext }}\left(\mathbf{x}_{i}, t\right)+\mathbf{e}^{\text {self }}\left(i, \mathbf{x}_{1 \ldots N}, \mathbf{p}_{1 \ldots N}\right), \\
\mathbf{b}_{i} & =\mathbf{b}^{\text {ext }}\left(\mathbf{x}_{i}, t\right)+\mathbf{b}^{\text {self }}\left(i, \mathbf{x}_{1 \ldots N}, \mathbf{p}_{1 \ldots N}\right) .
\end{aligned}
$$

The notation $\mathbf{x}_{1 \ldots N}$ is a shorthand for $\mathbf{x}_{1}, \ldots, \mathbf{x}_{N}$, and is used for other vectors analogously. The self field describes the field created by the collection of particles i.e. the source of the Coulomb repulsion. The external electromagnetic field (from magnets etc.), which can have an explicit dependence on time $t$, are in this model treated independent of the other particles.

\section{Constant time step integration method}

The Boris-Buneman integration scheme [10, pp. 58-63] solves the single particle equations of motion in electric and magnetic fields, and is a widely used orbit integrator in explicit PIC simulations and codes such as OPAL. The scheme is popular because it is simple to implement and because it gives second order accuracy while requiring only one force evaluation per step.

The integration method is similar to the leapfrog method (see e.g. [5]) as it offsets position and momentum by half a time step and updates them alternatingly. However, we have to deal with a non-separable system as the momentum derivative depends on the momentum itself in the magnetic force term. This requires special treatment to retain good properties like timesymmetry.

The classic derivation by Buneman and Boris is explained in [5] for the nonrelativistic case. The relativistic generalization is not difficult [10, pp. 356-357]. Here, we develop the method by an operator splitting approach [11, sec. II.5], directly working on the relativistic equations of motion. We will see that this yields exactly the same method. While this alternative derivation itself is not strictly necessary for this paper, it serves to introduce the operator-notation used to characterize the new integrator. In the operator-notation we make a simplifying assumption about the fields and look at only one particle, in order to allow the reader to quickly grasp the ideas. In the algorithms we will give all details how the integrators are implemented in our specific application.

We now look at a single particle and assume that the fields depend only on position $\mathbf{x}$, i.e. do not depend on $\mathbf{p}$ and are constant over time. Let the equations of motion be written as

$$
\frac{\mathrm{d}}{\mathrm{d} t}\left(\begin{array}{c}
\mathbf{x} \\
\mathbf{p}
\end{array}\right)=\left(\begin{array}{c}
\mathbf{f}_{X}(\mathbf{p}) \\
\mathbf{0}
\end{array}\right)+\left(\begin{array}{c}
\mathbf{0} \\
\mathbf{f}_{E}(\mathbf{x})
\end{array}\right)+\left(\begin{array}{c}
\mathbf{0} \\
\mathbf{f}_{B}(\mathbf{x}, \mathbf{p})
\end{array}\right),
$$

where

$$
\begin{aligned}
\mathbf{f}_{X}(\mathbf{p}) & =\mathbf{p} /(m \gamma(\mathbf{p})), \\
\mathbf{f}_{E}(\mathbf{x}) & =q \mathbf{e}(x), \\
\mathbf{f}_{B}(\mathbf{x}, \mathbf{p}) & =q \mathbf{p} \times \mathbf{b}(\mathbf{x}) /(m \gamma(\mathbf{p})) .
\end{aligned}
$$

For simplified systems where only one term of the right hand side of (8) exists we can make the following statements:

- If only $\mathbf{f}_{X}$ was present in the RHS, then

$$
\operatorname{Drift}_{h}:\left(\begin{array}{c}
\mathbf{x} \\
\mathbf{p}
\end{array}\right) \mapsto\left(\begin{array}{c}
\mathbf{x}+h \mathbf{f}_{X}(\mathbf{p}) \\
\mathbf{p}
\end{array}\right)
$$

would be the flow of the system, i.e. would integrate the system exactly.

- If only $\mathbf{f}_{E}$ was present in the RHS, then

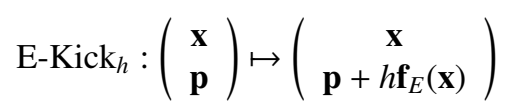

would be the flow of the system.

- If only $\mathbf{f}_{B}$ was present in the RHS, then

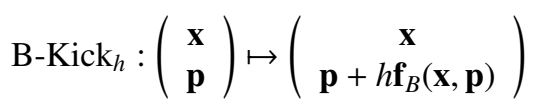

would be a numerical first-order approximation to the flow of the system (forward Euler method).

Having identified these parts, we construct a second-order integrator by using a suitably ordered composition of these mappings. By two nested applications of equation (5.9) [11, p. 49] (Combining Exact and Numerical Flows),

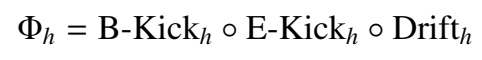

is a first-order integrator for the combined system. By composing the operator $\Phi_{h / 2}$ with its adjoint $\Phi_{h / 2}^{*}$ a second order integrator is obtained [11, p. 45],

$$
\begin{aligned}
& \mathrm{BB}_{h}=\Phi_{h / 2}^{*} \circ \Phi_{h / 2}=\left(\Phi_{-h / 2}\right)^{-1} \circ \Phi_{h / 2} \\
& =\operatorname{Drift}_{h / 2} \circ \\
& \underbrace{\text { E-Kick }_{h / 2} \circ\left(\mathrm{B}-\mathrm{Kick}_{-h / 2}\right)^{-1} \circ \mathrm{B} \mathrm{Kick}_{h / 2} \circ \mathrm{E}-\mathrm{Kick}_{h / 2}}_{\text {Kick }_{h}} \\
& \circ \operatorname{Drift}_{h / 2} \text {, }
\end{aligned}
$$

where the second equality recalls the definition of the adjoint. This is already the Boris-Buneman-method. However, the magnetic field kick in the middle

$$
\left(\begin{array}{c}
\mathbf{x}_{* *} \\
\mathbf{p}_{* *}
\end{array}\right)=\left(\text { B-Kick }_{-h / 2}\right)^{-1} \circ \text { B-Kick }_{h / 2}\left(\begin{array}{c}
\mathbf{x}_{*} \\
\mathbf{p}_{*}
\end{array}\right)
$$

is an implicit mapping. The p-component is

$$
\begin{array}{r}
\mathbf{p}_{* *}=\mathbf{p}_{*}+h q \mathbf{p}_{*} \times \mathbf{b}\left(\mathbf{x}_{*}\right) /\left(2 m \gamma\left(\mathbf{p}_{*}\right)\right)+ \\
h q \mathbf{p}_{* *} \times \mathbf{b}\left(\mathbf{x}_{* *}\right) /\left(2 m \gamma\left(\mathbf{p}_{* *}\right)\right),
\end{array}
$$


which is recognizable as the trapezoidal integration rule. Using that $\mathbf{x}_{*}=\mathbf{x}_{* *}$ and that the length of the momentum vector is invariant in the B-field rotation ( $\gamma$ stays constant), we have

$$
\frac{\mathbf{p}_{* *}-\mathbf{p}_{*}}{h}=q \frac{\mathbf{p}_{*}+\mathbf{p}_{* *}}{2 m \gamma\left(\mathbf{p}_{*}\right)} \times \mathbf{b}\left(\mathbf{x}_{*}\right) .
$$

Boris' important contribution is the way how $\mathbf{p}_{* *}$ can be calculated explicitly, thus making the whole method explicit.

In our application, the fields do not only depend on the position. But the change with respect to momentum and time are typically small within a time step, so the properties of the method still hold approximatively (assuming the time steps are not chosen too large). The method is implemented in OPAL as described in Algorithms 1, 2, and 3.
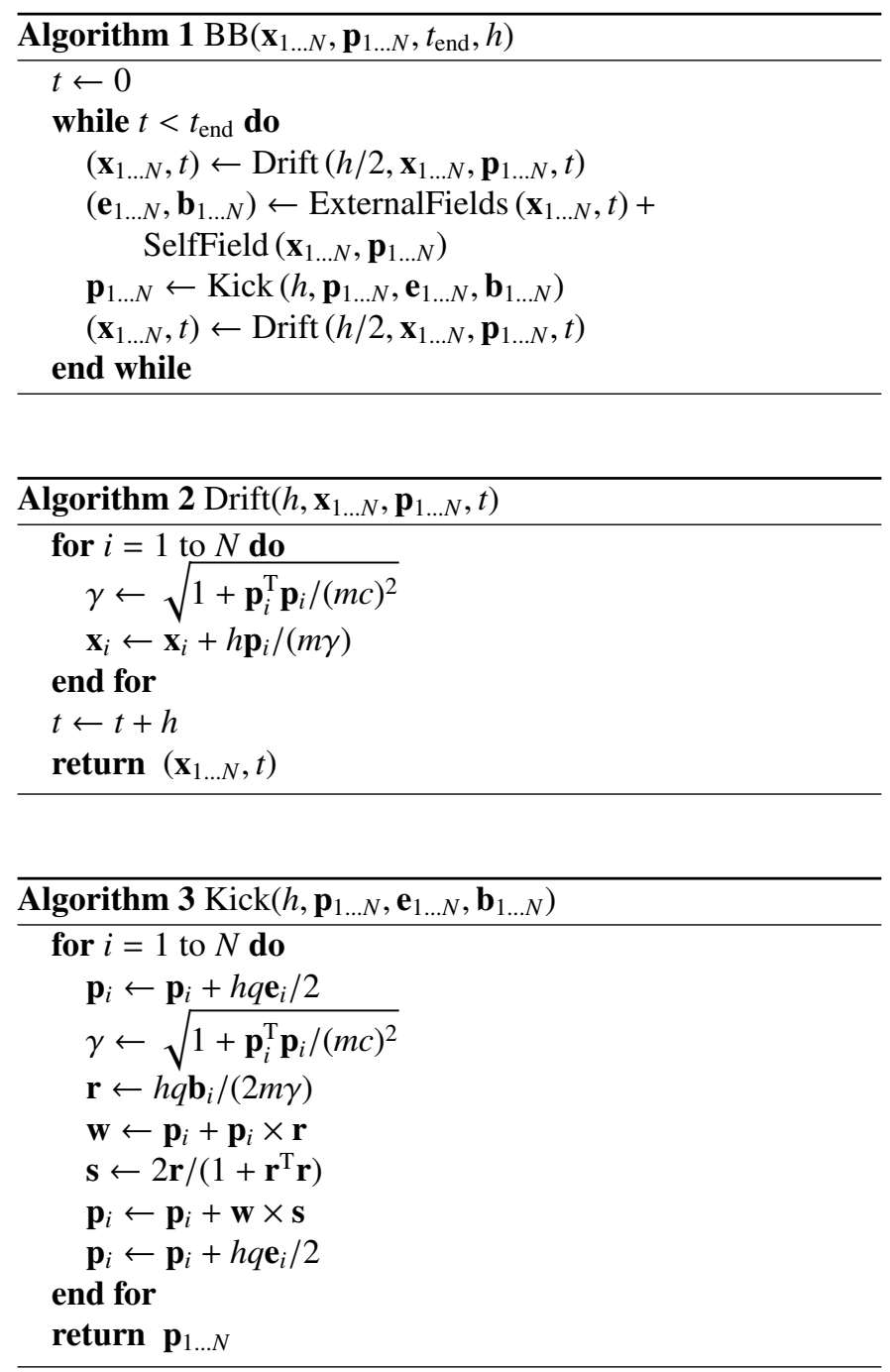

\section{Adaptive time step integration method}

\subsection{Multiple-time-stepping (MTS) variant}

Before discussing our adaptive step size variant of the Boris-Buneman integrator, we discuss a simple but powerful extension to the fixed time integration scheme from chapter 3 . We are following [11, Chapter VIII 4.1] and write the differential equation with a fast-slow splitting as

$$
\frac{\mathrm{d} \mathbf{p}}{\mathrm{d} t}=\mathbf{f}^{\mathrm{ext}}+\mathbf{f}^{\text {self }}
$$

$\mathbf{f}^{\text {self }}$ corresponds to the slow dynamics and is also the most expensive term to evaluate, namely the space charge forces. The fast dynamics is governed by $\mathbf{f}^{\text {ext }}$, and in our particular example, this term arises from fast varying external fields in an accelerator. For details see Algorithm 4.1 and Lemma 4.2 of [11, sec. VIII.4]. We state a multiple-time-stepping variant of the BorisBuneman integrator as

$$
\operatorname{MTS}_{h}^{m}=\operatorname{Kick}_{h / 2}^{\text {self }} \circ\left(\mathrm{BB}_{h / m}^{\text {ext }}\right)^{m} \circ \mathrm{Kick}_{h / 2}^{\text {self }}
$$

where the $m \geq 1$ substeps are defined by

$$
\mathrm{BB}_{h}^{\text {ext }}=\operatorname{Drift}_{h / 2} \circ \mathrm{Kick}_{h}^{\text {ext }} \circ \operatorname{Drift}_{h / 2} .
$$

The superscripts "ext" and "self" of the Kick-operator denote that only external and self-field forces, respectively, are used for the momentum update. The pseudo-code of the method is shown in Algorithm 4, which refers to Algorithm 5 and to already presented Algorithms 1, 2 and 3.
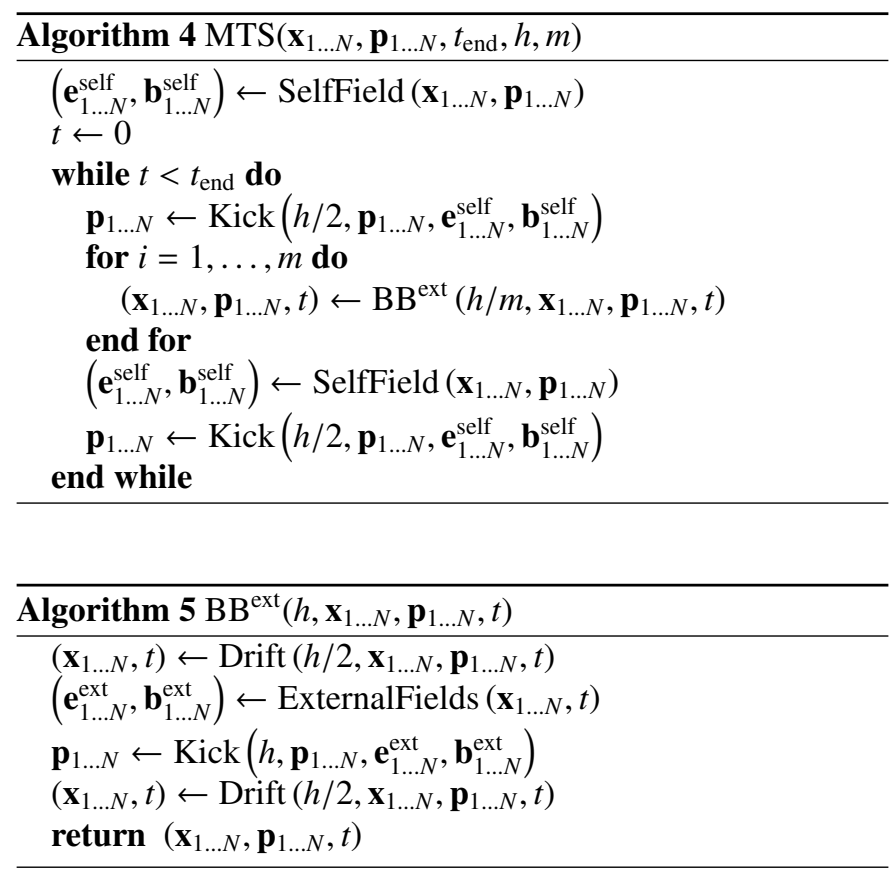

\subsection{Adaptive step size variant}

We explained in the introduction why geometric adaptive integration was used as the starting point in the design of our algorithm. Briefly, the simplicity of choosing the time step proportional to the system's state without having to deal with error estimates and step rejection was compelling, and the results of the previous work [5] motivated to further investigate this approach.

The concept of adaptive geometric integration is to look at a transformed system that evolves in a fictitious time $\tau$. The 
relation that connects the real time to it is called a Sundman transformation

$$
\frac{\mathrm{d} t}{\mathrm{~d} \tau}=g(\mathbf{z})
$$

The function $g$ determines the time rescaling and depends on the state of the system. It has to be chosen problem-dependent. The idea is that the rescaled system can be integrated with constant steps because the dynamics are regularized. When $g$ is large, $t(\tau)$ increases rapidly and we take large steps in real time. When $g$ is small, $t(\tau)$ increases slowly and we take small steps. Where the evolution of the system over $t$ was described with

$$
\frac{\mathrm{d} \mathbf{z}}{\mathrm{d} t}=\mathbf{f}(\mathbf{z}, t)
$$

the transformed equation with independent variable $\tau$ becomes, by the chain rule of differentiation,

$$
\frac{\mathrm{d} \mathbf{z}}{\mathrm{d} \tau}=\frac{\mathrm{d} \mathbf{z}}{\mathrm{d} t} \frac{\mathrm{d} t}{\mathrm{~d} \tau}=\mathbf{f}(\mathbf{z}, t) g(\mathbf{z})
$$

The curve $\mathbf{z}(\tau)$ is identical to $\mathbf{z}(t)$, but the trajectories are traversed at different velocities with respect to the independent variable. Constant steps taken in the fictitious time $\tau$ correspond to variable steps in the real time $t$, excluding the uninteresting case of a constant $g$. Multiple methods exist to solve such a transformed system.

Preserving time symmetry in the construction of the adaptive integration scheme is an important point for some applications, especially in few body settings like the Kepler problem. In [5] we noted that this symmetry is not important for our PIC application, as a consequence we do not put effort in enforcing this symmetry, favoring simplicity. The core concept we take over from the adaptive geometric integration technique is the choice of the time step proportional to some - cheap to evaluate - function of the current state.

How should we choose the function $g$ for our PIC application? Our inspiration came from the treatment of the Kepler problem. There the adaptive geometric integration technique demands smaller step sizes in situations where the force is large, i.e. when the two bodies are close to each other. In our case, we similarly want to demand smaller step sizes when particles are near to each other, i.e. when the beam volume is small and the space charge forces are large. Of course there is still a variety of ways how this can be done, especially because we have not only two bodies but a collection of particles. The key point here is: this approach to step size adaption is based solely on the strength of the space charge force, and not on the properties of the external fields. While this sounds limiting, the regularization of space charge solve frequency was the most evident opportunity to save computation cost in our application. It is certainly possible to extend the method with some sort of adaptivity for external fields, if required.

In order to determine $g$, for every particle, we compute its acceleration due to the space charge field with

$$
\mathbf{a}_{i}=\frac{\mathrm{d}^{2} \mathbf{x}_{i}}{\mathrm{~d} t^{2}}=\frac{1}{m \gamma_{i}}\left(\mathbf{f}_{i}^{\text {self }}-\mathbf{p}_{i} \frac{1}{m^{2} c^{2} \gamma_{i}^{2}} \mathbf{p}_{i}^{\mathrm{T}} \mathbf{f}_{i}^{\text {self }}\right),
$$

where $\mathbf{f}_{i}^{\text {self }}$ is the right hand side of (5) using only the self field contribution. The acceleration of largest magnitude among all particles is now related to the value of $g$ as

$$
g\left(\mathbf{p}_{1 \ldots N}, \mathbf{f}_{1 \ldots N}^{\text {self }}\right) \propto\left(\max _{i}\left\|\mathbf{a}_{i}\right\|\right)^{-\beta / 2} .
$$

Because only proportionality is important, one can leave out constants in the calculation of $g$. Section 5 will give an argument why $\beta=1.0$ is a good choice by analyzing adaptive integration of a one-dimensional model problem. In our experiments the overhead for calculating $g$ was negligible compared to the total time spent, but one can easily make this cheaper if required, e.g. by dropping the second term of the parenthesized part in (15) or by using a mean $\gamma$, avoiding per-particle square root operations.

In [5], we used a simpler version of $g$ which was based on the beam size directly. Also, there was only one time step. Here, we combine separate time steps for external and self fields together with variable step sizes for self fields. The MTS algorithm (see Alg. 4) is made adaptive ("AMTS") by

- using a variable outer time step $h$ adapted to space charge strength, and,

- using a variable $m \in \mathbb{N}$ such that the inner time step $h / m$ for external field integration is kept roughly at same size if possible.

The inner time step, of course, can never be higher than the outer time step. But as soon the outer time step exceeds some value, $m$ is increased to keep the external field step roughly at its original size. See Algorithm 6 for the full method. The initial step sizes must be specified as input.

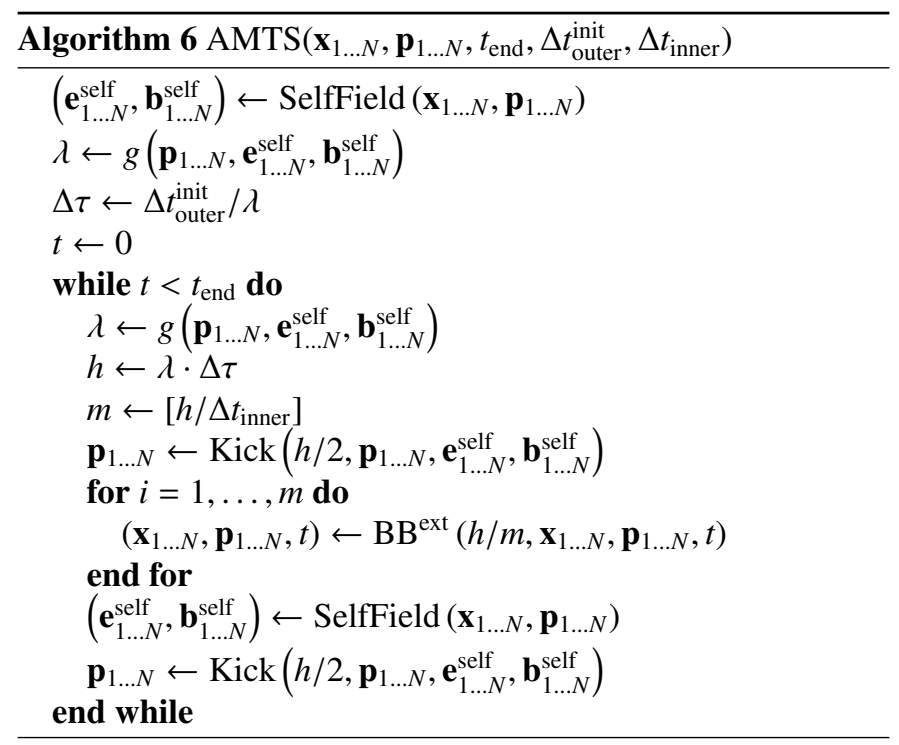

\section{Inverse square force integration}

\subsection{Model problem}

To better understand which adaptive strategy is optimal, a simple model problem is considered. We look at the motion 
of a single particle in one dimension, under the influence of a repulsive, Coulomb like force. For position $x$ and velocity $v$ of a single body, let the equations of motion be

$$
\begin{aligned}
& \frac{\mathrm{d} x}{\mathrm{~d} t}=v, \\
& \frac{\mathrm{d} v}{\mathrm{~d} t}=\frac{1}{x^{2}} .
\end{aligned}
$$

The force is proportional to the inverse square of the distance to the origin. Figure 1 shows the solution for the position and velocity as function of time. We can already 'guess' that a smaller

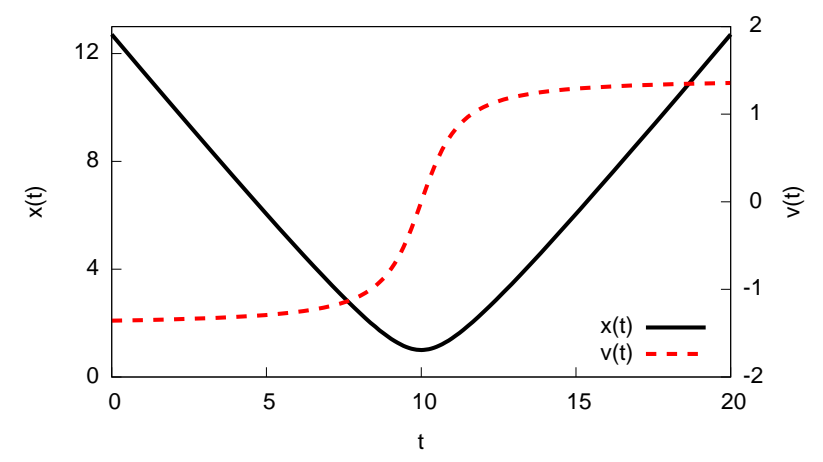

Figure 1: Solution of the 1D hard soft-wall collision problem. Shown are position $x(t)$ and velocity $v(t)$ as function of time.

time step near the minimum of $x$ (closest encounter) should be used. But we don't know how exactly the step size should depend on the situation. Therefore we investigate how the choice of the time rescaling function $g$ (from some class of candidates) influences the error of adaptive integration methods. The accuracy is measured by comparing to an analytical solution.

\subsection{Analytical solution}

This specific case allows an analytic treatment. The solution will not be explicit, but can be calculated by finding a root of some function.

Given the initial conditions $x_{0}>0$ and $v_{0}$, we determine $x_{\mathrm{L}}=x\left(t_{\mathrm{L}}\right)$ as the minimum of the curve $x(t)$. The energy $H(x, v)=v^{2} / 2+1 / x$ stays constant along the solution, and knowing $v\left(t_{\mathrm{L}}\right)=0$ leads to

$$
x_{\mathrm{L}}=\frac{2 x_{0}}{2+v_{0}^{2} x_{0}} .
$$

We can give the velocity (only positive solution used here) for any $x \geq x_{\mathrm{L}}$ by using the energy argument again:

$$
V(x)=\sqrt{2\left(\frac{1}{x_{\mathrm{L}}}-\frac{1}{x}\right)}
$$

Rearranging (18) using $\mathrm{d} v / \mathrm{d} t=\mathrm{d} v / \mathrm{d} x \cdot \mathrm{d} x / \mathrm{d} t=\mathrm{d} v / \mathrm{d} x \cdot v$ lets us integrate on both sides:

$$
\int_{x_{\mathrm{L}}}^{x} \frac{\mathrm{d} V(x)}{\mathrm{d} x} x^{2} \mathrm{~d} x=\int_{0}^{T(x)} \mathrm{d} t .
$$

Evaluating and simplifying gives us the (positive) time for the motion from $x_{\mathrm{L}}$ up to $x$ with

$$
T(x)=\sqrt{\frac{x_{\mathrm{L}}}{2}}\left(\sqrt{x\left(x-x_{\mathrm{L}}\right)}+x_{\mathrm{L}} \log \left(\frac{\sqrt{x-x_{\mathrm{L}}}+\sqrt{x}}{\sqrt{x_{\mathrm{L}}}}\right)\right) .
$$

The final solution is found in two steps. First, we compute the relative time needed to find the minimal $x$,

$$
t_{\mathrm{L}}= \begin{cases}-T\left(x_{\mathrm{L}}\right), & \text { if } v_{0}>0 \\ T\left(x_{\mathrm{L}}\right), & \text { otherwise }\end{cases}
$$

then we compute the final condition from

$$
\begin{aligned}
& x(t)= \begin{cases}T^{-1}\left(t-t_{\mathrm{L}}\right), & \text { if } t>t_{\mathrm{L}}, \\
T^{-1}\left(t_{\mathrm{L}}-t\right), & \text { otherwise, }\end{cases} \\
& v(t)= \begin{cases}V(x(t)), & \text { if } t>t_{\mathrm{L}}, \\
-V(x(t)), & \text { otherwise. }\end{cases}
\end{aligned}
$$

To compute $T^{-1}(t)$, we find the root of $T(x)-t$ by bisecting in the interval $\left[x_{\mathrm{L}}, x_{\mathrm{L}}+t^{2} / x_{\mathrm{L}}^{2}\right]$.

\subsection{Experiment and findings}

We already know that the numerical integration efficiency can be increased by concentrating effort on parts where the force is large, see e.g. the Kepler problem in [5]. The considered adaptive methods employ a Sundman transformation of the form

$$
\frac{\mathrm{d} t}{\mathrm{~d} \tau}=g(x)=x^{\beta}=\left(\frac{\mathrm{d}^{2} x}{\mathrm{~d} t^{2}}\right)^{-\beta / 2}
$$

applied to the system 177 - 18 . In other words, we aim at making the variable timestep proportional to $g$. Of course there are other possibilities how to choose the transformation, but the dependeny on solely $x$ means that the timestep will be a function of the magnitude of the acceleration/force. For problems with many bodies (e.g. our PIC simulation), we then can use the maximal acceleration/force for the choice of a global timestep. The goal is now to look more closely at the exponent $\beta$ and ask for an optimal value.

In the following, we integrate three different scenarios until $t_{\text {end }}=20$. The initial conditions are chosen such that the lowest point is reached at $t_{\mathrm{L}}=10$, i.e. $x_{0}=T^{-1}(10)$ and $v_{0}=-V\left(x_{0}\right)$. The scenarios differ in the energy level $H$. A higher energy level means the magnitude of the initial velocity is larger and the minima appear at a lower $x_{\mathrm{L}}$. For example, the energy level $H=10^{0}$ corresponds to Fig. 1 where $x_{\mathrm{L}}=1$. The adaptive Verlet method (see references in [5]) is used for integration. Since there are multiple variants of this method that differ in operator splitting and timestep adaption, we give Algorithm 7 to remove ambiguity and to allow easy verification of the results. It can be expected that the essence of the result (what is optimal $\beta$ ) also holds for other similar adaptive methods. The initial timestep was chosen such that each run reached $t_{\text {end }}$ in 1000 steps.

Figure 2 shows that exponent $\beta=1$ is a good choice, where the difference to other exponents gets pronounced at higher energy levels. We also experimented with other variants of the 


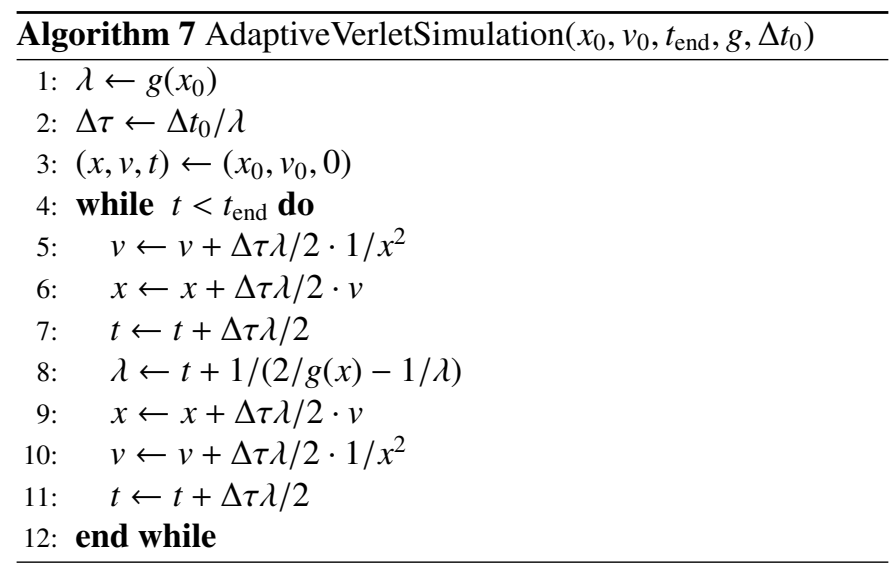

adaptive Verlet method and a symplectic adaptive method (see [5] p. 23]). They all showed similar behaviour.

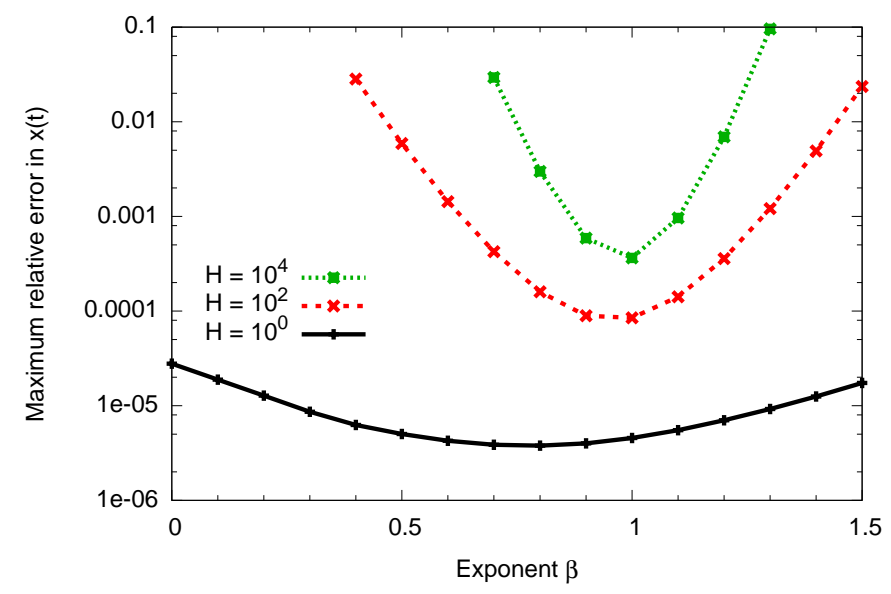

Figure 2: Error of adaptive time step integration depending on exponent choice in Sundman transformation. Missong points in the figure indicate that the respective simulation runs broke down, e.g. because of negative $x$ values produced during integration.

We see a discrepancy compared to what [12] considers as optimal in this so-called hard soft-wall problem and note that in [12] not only Coulomb potentials but a whole class of $1 / x^{\alpha}$ potentials are studied. If we insert $\alpha=1$ for our case, the choice $\beta=3 / 2$ is proposed. Their findings are based on a scale invariance argument and they verify the result with experiments for larger $\alpha$ than in our case. It could be that for the specific case $\alpha=1$, the general result is too approximative. In our opinion, authors previously using $\beta=3 / 2$ should review this choice.

\section{Applications}

The presented algorithms were implemented and tested in the OPAL accelerator simulation framework [1]. In the first experiment, we compare AMTS to MTS, and in the second experiment, we compare MTS to the existing Boris-Buneman integrator. All simulations were performed with small resolution (few thousand particles and small PIC mesh resolution) on a laptop computer. But it can be expected that the results carry over to large-scale simulations, where of course the absolute gain in computation time is higher.

\subsection{Photoinjector}

We simulate a beam for the first half meter in a photoinjector. This is an example in which space charge forces are important because of the low beam energy, and where the magntiude of the space charge forces changes considerably during the simulation. See Fig. 3 for the energy curve, and Fig. 4 for the development of the beam size.

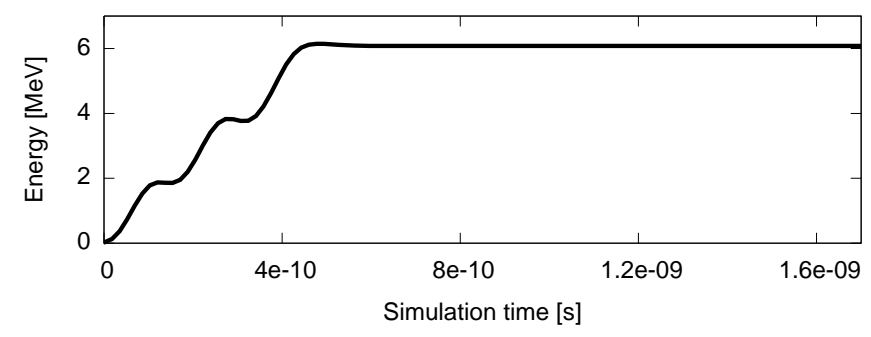

Figure 3: Mean particle energy in the photoinjector scenario.

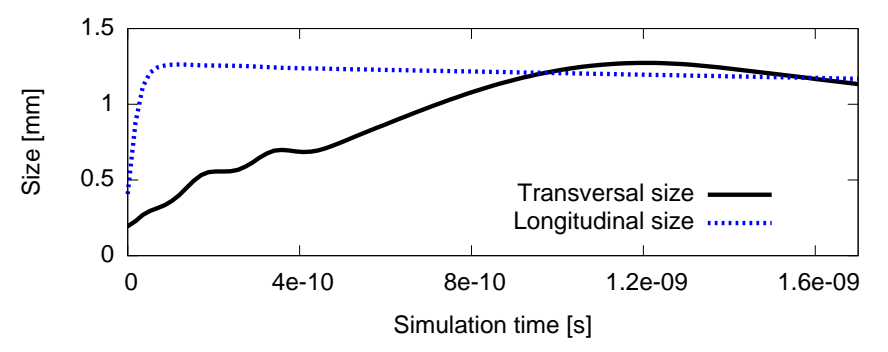

Figure 4: Transversal and longitudinal root-mean-square size of the beam for the photoinjector.

We show that the adaptive step size variant of the multipletime-stepping integrator (AMTS) is more efficient than the constant MTS integrator. To allow a comparison of the different integration strategies with respect to space charge effects, we want to treat external field integration as much as possible equally among all runs. In MTS the inner time step is fixed to $\Delta t_{\text {inner }}=5 \cdot 10^{-13}$, and the outer step size is a multiple of it. In AMTS, as the outer time step increases, more substeps are used

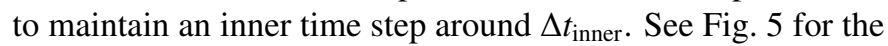
time step choice of an adaptive run. The strong variation in the space charge forces leads to a change in the (outer) time step of more than a factor 100 .

In Fig. 6 we give a comparison of the error made in transverse root-mean-square emittance (a measure of the phase space volume) for different amount of work spent on self field calculations. The errors are with respect to a reference solution obtained using the MTS integrator with $m=1$ and a step size of $5 \cdot 10^{-13}$. AMTS reaches a given error with drastically fewer self field calculations. 


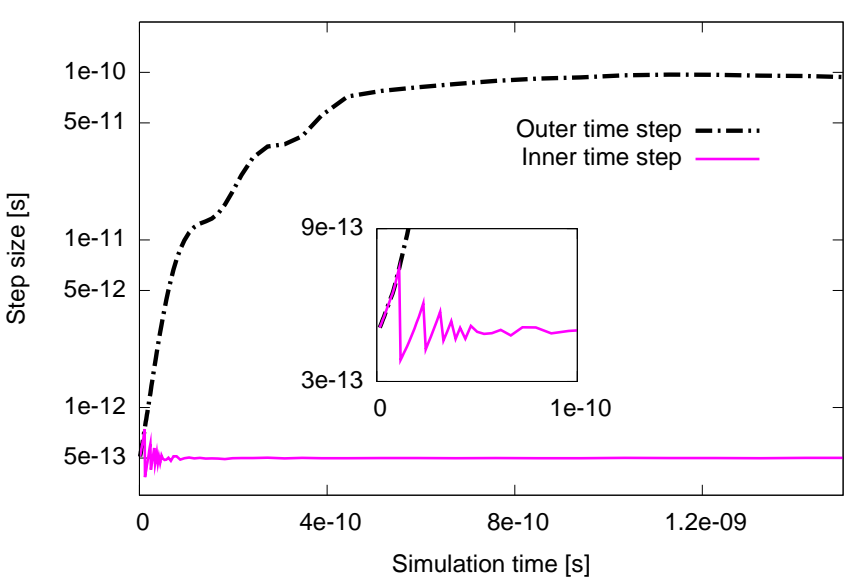

Figure 5: Time step choice of an AMTS run in the photoinjector simulation. Because the method requires an integer number of substeps per outer step, the inner time step slightly fluctuates around the desired value.

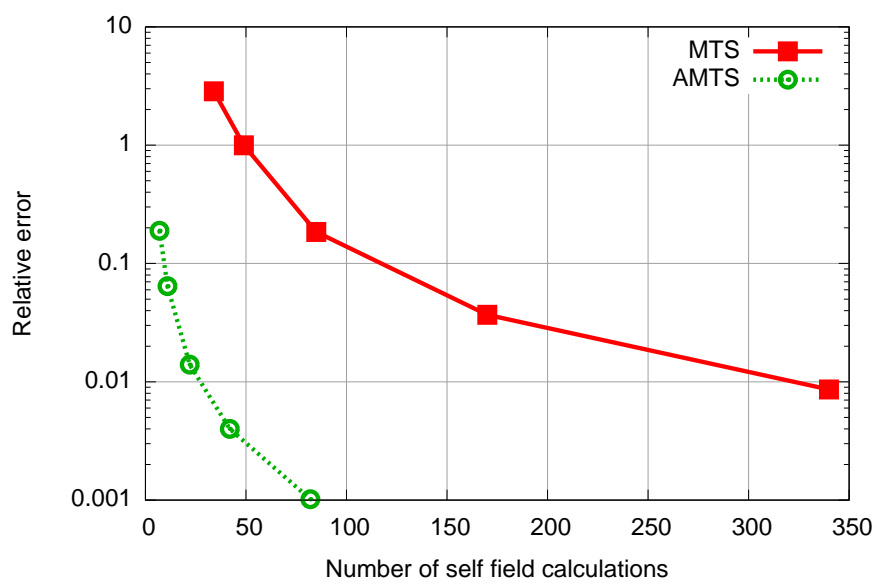

Figure 6: Time integration error in the transverse root-mean-square emittance for the photoinjector simulation. Errors are with respect to a reference solution obtained with MTS using 3400 self field calculations.

In Fig. 7 we compare different choices of the $g$-function, which determines how the variable time step is chosen. Although all choices are far better than a constant step, we see that our choice with $\beta=1.0$, derived with the $1 \mathrm{D}$ model, gives slightly better results than the other considered possibilities. It is also better than the choice from experiments reported in [5], which used a much simpler adaptation criterion, namely the beam size.

Note that the actual time spent on these simulations is dominated by the external field integration, because the inner time step is kept very small. The reason why we choose the inner time step unpractically small is that the work spent for external field integration should be kept constant among the runs, such that the comparison using the number of self field calculations makes sense. For practical simulations, the inner time step can be chosen larger hence for the same accuracy, AMTS yields a significant reduction in time-to-solution compared to the other

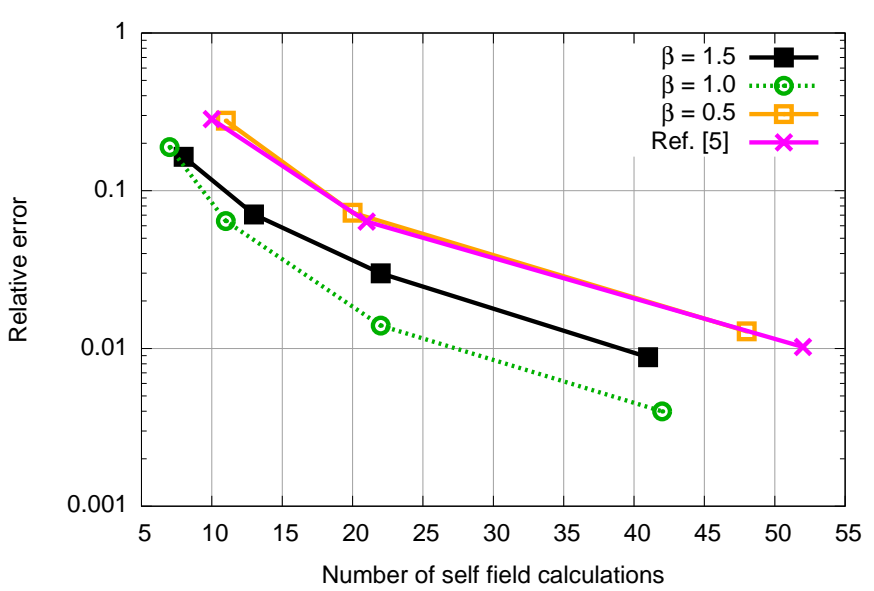

Figure 7: Comparison of different choices of the $g$-function for the AMTS integrator.

integrators.

\subsection{Cyclotron}

The simulation library OPAL [1] has an own component for the simulation of cyclotrons. This component was recently enhanced with support for neighboring bunch effects [13], increasing significantly the number of space charge calculations in a simulation. For this comparison, we simulate the first 10 turns of the PSI $590 \mathrm{MeV}$ ring cyclotron, with an initial particle energy of $72 \mathrm{MeV}$. First, we had to recognize that a variable step size (AMTS) brings no visible benefit over the plain MTS algorithm. This is understandable as the variation in space charge forces is far smaller than in the photoinjector case. Also, the influence of space charge is smaller because the beam energy is higher. Therefore, we use this case to report about the difference between the original integrator and the MTS variant.

We now want to observe how close a run with reduced space charge solve frequency (MTS with $m>1$ ) comes to the original solution, and how much time we save. As measure, we take again transversal RMS emittance. Relative errors are calculated at five points per turn, and then the maximum of these errors counts as overall error. The timings are not meant to be a precise profiling, but should give a hint how much time is roughly saved. In general, the savings depend mainly on the fraction of space charge solve cost to overall cost of the simulation, beside further details in the implementation. See Table 1 for the results. Space charge has certainly an influence in this scenario, as the error of the solution without simulating space charge is not small. The surprising fact is that a drastically reduced space charge solve frequency is still very accurate, but saves a lot of time.

The existing Boris-Buneman implementation in OPAL (without MTS extension) had an option to calculate the space charge forces only every $n$-th step, and then reusing the old forces in the next $n-1$ time steps. This was a first step towards reducing computation cost. In this context it is interesting to see how this first approach compares to the new MTS method. In Table 2 we 


\begin{tabular}{lll}
\hline SC solve frequency & Error in $\%$ & CPU time (seconds) \\
\hline$m=1$ & 0 & 524 \\
$m=2$ & $3.35 \cdot 10^{-6}$ & 357 \\
$m=4$ & $1.52 \cdot 10^{-5}$ & 273 \\
$m=10$ & $1.15 \cdot 10^{-4}$ & 222 \\
$m=20$ & $5.13 \cdot 10^{-4}$ & 207 \\
$m=100$ & $8.89 \cdot 10^{-2}$ & 193 \\
No space charge & 12.0 & 190 \\
\hline
\end{tabular}

Table 1: Maximal relative error in transversal RMS emittance and timings of MTS integrator in the ring cyclotron simulation. Increasing the MTS substep number $m$ (see 10) reduces the number of self field computations, thus saving time while introducing some deviation to the solution with full solve frequency $(m=1)$. The solution with no space charge effects modeled is given as comparison.

give errors and timings for this older approach. Again, as the timings depend on implementation details, only the big picture is important here. It is apparent that this, without prejustice seemingly reasonable scheme (reuse something that changes slowly), builds up a large error even with low values of $n$, and was therefore rarely used. It seems that reusing of old forces introduces an asymmetry which hurts the accuracy, and a proper MTS implementation should be used instead.

\begin{tabular}{lll}
\hline SC solve frequency & Error in \% & CPU time (seconds) \\
\hline$n=1$ & 0 & 473 \\
$n=2$ & $1.01 \cdot 10^{-1}$ & 344 \\
$n=4$ & $2.62 \cdot 10^{-1}$ & 281 \\
$n=10$ & 1.45 & 242 \\
$n=20$ & 5.46 & 230 \\
$n=100$ & 15.4 & 220 \\
No space charge & 12.0 & 197 \\
\hline
\end{tabular}

Table 2: Maximal relative error in transversal RMS emittance and timings of modified Boris-Buneman integrator in the ring cyclotron simulation. Calculating the self field only every $n$-th step reduces the number of self field computations, thus saving time while introducing some deviation to the solution with full solve frequency $(n=1)$. The solution with no space charge effects modeled is given as comparison.

\section{Conclusions}

We presented two time integration schemes that enhance the standard Boris-Buneman algotitm by adapting the frequency of self field calculations. The usability was demonstrated within the OPAL particle accelerator framework, but the methods could be easily applied to similar problems.

The multiple time stepping (MTS) extension allows to compute the self fields less frequently, by a factor which has to be defined beforehand. For many scenarios where space charge has a visible contribution but is not dominant, this method can save considerable computation time with only negligibly changing the solution. While the multiple time stepping strategy is not new, we have not seen it applied in this context. Initially intended to be an intermediate algorithm only to derive the variable step size variant, MTS itself turned out to be of practi- cal relevance, as its implementation incurs hardly any overhead while the performance gain is substantial.

The adaptive multiple time stepping (AMTS) algorithm introduces a variable step size integrator. Variable step sizes are most beneficial over MTS in space charge dominated (low energy) simulations. If space charge forces change considerably, like in gun simulations, AMTS shows its strengths. While previous work indicated that variable step sizes can be useful in such cases, this work gives further insight on how the step size should be adapted for good results. The foundation on MTS allows to have two individual time steps, an outer one that can be adapted to self field situation, and an inner one that can be kept small for external fields. The implementation is more complicated than for MTS. However, we have shown that important problems exist for which this additional effort pays off.

\section{Acknowledgements}

We are grateful to A. Fallahi for discussions related to the analysis of the single particle model problem, to $\mathrm{S}$. Wei for performing first practical tests with the MTS method, and to J. Conrad for financial support.

\section{References}

\section{References}

[1] A. Adelmann, C. Kraus, Y. I. et.al, The OPAL (Object Oriented Parallel Accelerator Library) Framework, Technical Report PSI-PR-08-02, Paul Scherrer Institut, 2008-2010. http: //amas .web.psi .ch/docs/opal/ opal_user_guide.pdf

[2] S. K. Agarwalla, J. M. Conrad, M. Shaevitz, Short-baseline Neutrino Oscillation Waves in Ultra-large Liquid Scintillator Detectors, arXiv 1105.4984, hep-ph (2011).

[3] M. Abs, A. Adelmann, et al., Multimegawatt DAE $\delta$ ALUS Cyclotrons for Neutrino Physics, arXiv 1207.4895, physics.acc-ph (2012).

[4] A. Bungau, A. Adelmann, et al., An Electron Antineutrino Disappearance Search Using High-Rate 8Li Production and Decay, Phys.Rev.Lett. 109 (2012) 141802.

[5] M. Toggweiler, An adaptive time integration method for more efficient simulation of particle accelerators, Master's thesis, ETH Zurich, 2011. http://e-collection.library.ethz.ch/list/author? author=Toggweiler\%2C+Matthias

[6] C. W. Gear, Numerical initial value problems in ordinary differential equations, Prentice-Hall, Englewood Cliffs, N.J, 1971.

[7] L. D. Landau, E. M. Lifshitz, Electrodynamics of Continuous Media, Pergamon, Oxford, 2nd edition, 1984.

[8] J. Qiang, R. D. Ryne, Parallel 3D Poisson solver for a charged beam in a conducting pipe, Comput. Phys. Commun. 138 (2001) 18-28.

[9] J. Qiang, R. L. Gluckstern, Three-dimensional Poisson solver for a charged beam with large aspect ratio in a conducting pipe, Comput. Phys. Commun. 160 (2004) 120-128.

[10] C. K. Birdsall, A. B. Langdon, Plasma Physics via Computer Simulation, McGraw-Hill, 1985.

[11] E. Hairer, C. Lubich, G. Wanner, Geometric Numerical Integration: Structure-Preserving Algorithms for Ordinary Differential Equations, Springer, Berlin, 2006.

[12] S. Bond, B. Leimkuhler, Time-transformations for reversible variable stepsize integration, Numer. Algorithms 19 (1998) 55-71.

[13] J. Yang, A. Adelmann, M. Humbel, M. Seidel, T. Zhang, Beam Dynamics in High Intensity Cyclotrons Including Neighboring Bunch Effects: Model, Implementation and Application, Phys. Rev. ST Accel. Beams 13 (2010) 064201. 